\title{
PORT-BASED CONTROL OF A COMPASS-GAIT BIPEDAL ROBOT
}

\author{
Vincent Duindam Stefano Stramigioli
}

\author{
Control Laboratory, Fac. of EEMCS, University of Twente \\ P.O.Box 217, 7500 AE Enschede, Netherlands \\ v.duindam@ieee.org s.stramigioli@ieee.org
}

\begin{abstract}
We examine the control problem of curve-tracking for fully-actuated mechanical systems, applied to walking robots. First, we discuss earlier results on curve-tracking using a change of coordinates to split the kinetic energy in a desired and an undesired part. Then, we apply and extend these results to the case of a compass-gait biped, and show in a simulation how its robustness against surface height variations can be increased. Copyright (c)2005 IFAC
\end{abstract}

Keywords: port-Hamiltonian systems, passive compensation, legged locomotion.

\section{INTRODUCTION}

Passive walkers, as introduced in (McGeer, 1989), have taken an important place in research on walking robots: seemingly simple robots are able to walk down slopes in perfectly natural-looking motion, even though they are completely uncontrolled and only get their energy from gravity.

Unfortunately, these types of walking robots are not very robust against disturbances. Experiments (Collins et al., 2001) have shown that careful engineering and tuning is necessary to obtain a stable gait, and also theoretical research has shown that the limit cycles often only have a narrow region of attraction.

In this paper, we discuss a method for adding a controller to the robot that does not disturb the nominal passive walking cycle but provides a corrective action only when the robot deviates from the nominal behavior (e.g. because of a disturbance). The controller is based on the general results of (Duindam and Stramigioli, 2005) but slightly adapted to the case of a walking robot. It consists of a cascade port-interconnection of several power-continuous sub-controllers for specific subgoals, plus a passive energy-shaping controller.
The general control goal is for a mechanical system to move asymptotically along a certain predescribed subspace of the configuration space. For the walking robot, we take as this desired subspace the nominal path (a one-dimensional subspace) that it would follow during uncontrolled passive walking.

The control idea in this paper is related to the Passive Velocity Field Control (PVFC) strategy described in (Li and Horowitz, 1995; $\mathrm{Li}$ and Horowitz, 1999), but the main differences are (1) PVFC uses temporal energy storage in the form of a virtual flywheel whereas our approach is powercontinuous, and (2) PVFC aims to have the velocity equal to a fixed multiple of a vector field, while we just wish to have the velocity in the direction of the vector field; the magnitude is not important.

The remainder of this paper is structured as follows. Section 2 describes the framework of portHamiltonian systems which is used throughout the paper. Section 3 then summarizes earlier results in port-based control of fully-actuated mechanical systems. Section 4 adapts and applies these results to the case of a simple bipedal robot and shows how robustness is increased. Finally, 
Section 5 summarizes the main conclusions of the paper and presents an outlook on future work.

\section{PORT-HAMILTONIAN SYSTEMS}

A general explicit port-Hamiltonian system is a dynamical system that can be represented by a set of differential equations of the following form

$$
\left\{\begin{array}{l}
\dot{x}=(J(x)-R(x)) \frac{\partial H(x)}{\partial x}+g(x) u \\
y=g^{T}(x) \frac{\partial H(x)}{\partial x}+(K(x)+S(x)) u
\end{array}\right.
$$

in which $x \in \mathcal{X}$ is the state, $H: \mathcal{X} \rightarrow \mathbb{R}$ is the (differentiable) energy function, $J(x)$ and $K(x)$ are skew-symmetric matrices (to model powercontinuous elements), $R(x)$ and $S(x)$ are positive semi-definite matrices (to model dissipative elements), and $(u, y) \in \mathcal{U} \times \mathcal{U}^{*}$ is the port through which the system can interact with e.g. a controller. For systems of this form it is straightforward to show that $\dot{H} \leq u^{T} y$, i.e., the system is passive with respect to the port $(u, y)$ with storage function $H$. Several generalizations for this kind of systems exist, e.g. more general implicit formulations, and we refer the interested reader to (van der Schaft, 2000) and (Blankenstein, 2000).

In this paper, we consider the subclass of mechanical systems (with $H$ the mechanical energy) and we start from a conservative simple mechanical system (a system for which the total energy is the sum of kinetic and potential energy). The dynamics of such a system can be described in port-Hamiltonian form as

$$
\begin{aligned}
\frac{d}{d t}\left[\begin{array}{l}
q \\
p
\end{array}\right] & =\left[\begin{array}{cc}
0 & I \\
-I & 0
\end{array}\right]\left[\begin{array}{l}
\frac{\partial H}{\partial q} \\
\frac{\partial H}{\partial p}
\end{array}\right]+\left[\begin{array}{l}
0 \\
B
\end{array}\right] u \\
y & =\left[\begin{array}{ll}
0 & B^{T}
\end{array}\right]\left[\begin{array}{l}
\frac{\partial H}{\partial q} \\
\frac{\partial H}{\partial p}
\end{array}\right]
\end{aligned}
$$

where $(q, p)$ are the generalized positions and momenta, i.e., the canonical coordinates on the cotangent bundle, and $H$ equals

$$
H(q, p)=\frac{1}{2} p^{T} M^{-1}(q) p+V(q) .
$$

The first term of $H$ is the kinetic energy (with $M(q)$ the symmetric positive-definite mass matrix), the second term is the potential energy. Systems described in these coordinates $(q, p)$ with $J$ as shown are called symplectic systems.

The mass matrix $M$ defines a metric on the tangent bundle (the space of all velocities) and hence an inner product between two vectors $v$ and $w$ as $\langle v, w\rangle=v^{T} M w$. Similarly, $M$ defines an inner product on the cotangent bundle (the space of all momenta) acting on covectors $p$ and $r$ as $\langle p, r\rangle=p^{T} M^{-1} r$.

\section{PORT-BASED CURVE TRACKING}

Before turning to the case of walking robots, we first look at a general fully-actuated simple mechanical system described by (2) with $B$ invertible. As stated in Section 1, the control goal is to make the system converge to a motion along some pre-described subspace $Q_{d} \subset Q$ (with $Q$ the configuration manifold), for example a curve (onedimensional subspace). In this section, we generalize the results of (Duindam and Stramigioli, 2005) to the case of a multi-dimensional subspace.

Instead of immediately trying to tackle the problem of convergence to $Q_{d}$, we first relax the control goal as follows: in addition to $Q_{d}$, we define a family of non-intersecting submanifolds, one through each point of $Q$, in the form of a smooth distribution on $Q$ (a set of non-zero independent vector fields). This distribution is represented by a smoothly varying $n \times m$ matrix $\mathcal{D}(q)$, the columns of which are the vector fields ${ }^{1}$.

The initial goal is now for the system to converge to $\mathcal{D}$, i.e., to align the velocity of the system with the distribution and keep it aligned. Convergence to the $Q_{d}$ can then be obtained by either choosing the distribution to converge automatically ( $\mathrm{Li}$ and Horowitz, 1999), or by adding a suitably chosen potential field (Duindam and Stramigioli, 2005).

\subsection{Change of Coordinates}

Before designing the controller, we first represent the system in different coordinates to be able to separate desired motions (motion along the distribution) from undesired motion (motion perpendicular to the distribution). We keep the same coordinates $q$ for the position but replace the momenta $p$ by $h(q) \alpha$, where $\alpha$ are the new coordinates for the momentum and $h(q)$ is structured as

$$
h:=\left[h_{d} h_{u}\right]:=\left[\left(h_{1} \cdots h_{m}\right)\left(h_{m+1} \cdots h_{n}\right)\right]
$$

and satisfies the following properties:

(1) the matrix $h(q)$ is invertible and varies smoothly with $q$, i.e., it defines a proper change of coordinates according to

$$
\begin{aligned}
p & =h(q) \alpha \\
\alpha & =h^{-1}(q) p
\end{aligned}
$$

\footnotetext{
1 This automatically implies that we will take a local approach, since the topology of the configuration space as well as the shape of the specified desired submanifold can make it impossible to define such a distribution globally (e.g. on $\mathbb{S}^{2 n}$ this is the famous 'hairy-ball theorem').
} 
(2) the last $n-m$ columns of $h$ are orthogonal to $\mathcal{D}$, i.e. $h_{u}^{T}(q) \mathcal{D}(q)=0$.

(3) the metric induced by $M(q)$ and $h(q)$ is structured as

$$
h^{T} M^{-1} h=\left[\begin{array}{cc}
h_{d}^{T} M^{-1} h_{d} & 0 \\
0 & h_{u}^{T} M^{-1} h_{u}
\end{array}\right]
$$

and is independent of $q$.

These properties basically mean that the first $m$ columns of $h$ describe the desired motion along the distribution $\mathcal{D}$, that the last $n-m$ columns of $h$ are orthogonal (in the metric $M^{-1}$ ) to the first $m$ columns and describe the undesired motion, and that all columns of $h$ have constant norm and relative angle (in $M^{-1}$ ).

The energy of the system can now be expressed in the new coordinates as

$$
\begin{aligned}
& \bar{H}(q, \alpha):=H(q, h \alpha)=\frac{1}{2} \alpha^{T} h^{T} M^{-1} h \alpha+V \\
& \quad=\frac{1}{2} \alpha_{d}^{T} h_{d}^{T} M^{-1} h_{d} \alpha_{d}+\frac{1}{2} \alpha_{u}^{T} h_{u}^{T} M^{-1} h_{u} \alpha_{u}+V
\end{aligned}
$$

where $\alpha^{T}=\left[\begin{array}{ll}\alpha_{d}^{T} & \alpha_{u}^{T}\end{array}\right]$ is split similarly to $h$. This shows that the new coordinates separate the kinetic energy in the desired direction (depending only on $\alpha_{d}$ ) from the kinetic energy in the undesired direction (depending only on $\alpha_{u}$ ) and the potential energy (depending on $q$ ).

Given a certain choice of $h$ satisfying the criteria, we can rewrite the dynamic equations in the new coordinates as presented in the following theorem.

Theorem 1. A mechanical system defined by (2) with coordinate transformation defined by $h$ as before can be written as

$$
\begin{gathered}
\frac{d}{d t}\left[\begin{array}{c}
q \\
\alpha
\end{array}\right]=\bar{J}\left[\begin{array}{l}
\frac{\partial \bar{H}}{\partial q} \\
\frac{\partial \bar{H}}{\partial \alpha}
\end{array}\right]+\left[\begin{array}{c}
0 \\
h^{-1} B
\end{array}\right] u \\
y=\left[\begin{array}{ll}
0 & B^{T} h^{-T}
\end{array}\right]\left[\begin{array}{c}
\frac{\partial \bar{H}}{\partial q} \\
\frac{\partial \bar{H}}{\partial \alpha}
\end{array}\right]
\end{gathered}
$$

where

$$
\bar{J}:=\left[\begin{array}{cc}
0 & h^{-T} \\
-h^{-1} & h^{-1}\left(\frac{\partial^{T}(h \alpha)}{\partial q}-\frac{\partial(h \alpha)}{\partial q}\right) h^{-T}
\end{array}\right]
$$

and $\bar{H}(q, \alpha)=\frac{1}{2} \alpha^{T} h^{T}(q) M^{-1}(q) h(q) \alpha+V(q)$.

PROOF. See (Duindam and Stramigioli, 2005).

Fig. 1 shows a schematic block diagram of the plant in new coordinates (together with the controller from the following section). The energy has been split in a desired and an undesired part and the block labeled 'power-continuous interconnection' describes the possible flows of energy in the system, as defined by $\bar{J}$ and the input-output mappings.

\subsection{Power-Continuous Asymptotic Curve Tracking}

We now propose a port-based controller that makes the system move along integral curves of the distribution $\mathcal{D}$.

Theorem 2. Given the mechanical system (2) or in transformed coordinates (5), define the following sub-controllers (see also Fig. 1):

- Input transformation:

$$
\left[\begin{array}{l}
u \\
\hat{y} \\
\bar{y}
\end{array}\right]=\left[\begin{array}{ccc}
0 & B^{-1} & B^{-1} \\
-B^{-T} & 0 & 0 \\
-B^{-T} & 0 & 0
\end{array}\right]\left[\begin{array}{c}
-y \\
\hat{u} \\
\bar{u}
\end{array}\right]
$$

- Energy storage:

$$
\left\{\begin{array}{l}
\frac{d}{d t} \hat{q}=\hat{y} \\
-\hat{u}=\frac{\partial \hat{V}}{\partial \hat{q}}
\end{array}\right.
$$

- Decoupling controller:

$$
\left[\begin{array}{c}
\bar{u} \\
\tilde{y}_{d} \\
\tilde{y}_{u}
\end{array}\right]=\left[\begin{array}{ccc}
X & h_{d} & h_{u} \\
-h_{d}^{T} & 0 & 0 \\
-h_{u}^{T} & 0 & 0
\end{array}\right]\left[\begin{array}{c}
-\bar{y} \\
\tilde{u}_{d} \\
\tilde{u}_{u}
\end{array}\right]
$$

with

$$
X:=\left(\frac{\partial h \alpha}{\partial q}-\frac{\partial^{T} h \alpha}{\partial q}\right)+h\left[\begin{array}{cc}
S_{d} & 0 \\
0 & S_{u}
\end{array}\right] h^{T}
$$

where $S_{d}$ and $S_{u}$ are arbitrary skew-symmetric matrices of size $m \times m$ and $(n-m) \times(n-m)$, respectively.

- Convergence controller:

$$
\left[\begin{array}{l}
\tilde{u}_{d} \\
\tilde{u}_{u}
\end{array}\right]=\left[\begin{array}{cc}
0 & -a \tilde{y}_{d} \tilde{y}_{u}^{T} \\
a \tilde{y}_{u} \tilde{y}_{d}^{T} & 0
\end{array}\right]\left[\begin{array}{l}
-\tilde{y}_{d} \\
-\tilde{y}_{u}
\end{array}\right]
$$

If we furthermore choose $\hat{V}(q)=-V(q)$ and take initial conditions such that $\hat{q}(0)=q(0)$, then the cascade port-interconnection of the subcontrollers (6)-(10) to the plant results in asymptotic convergence to motion along $\mathcal{D}$.

PROOF. We give a quick intuitive sketch of the proof of the theorem; more details can be found in (Duindam and Stramigioli, 2005).

First, it can be seen that, given the initial condition that $\hat{q}(0)=q(0), \hat{q}$ is equal to $q$ for all $t \geq 0$. By choosing the controller energy $\hat{V}$ as the negative of the system's potential energy, the effect of the potential energy is effectively canceled out. This part of the controller is simply a version of classical energy shaping.

Second, under the conditions above, we can write the equations for the desired and undesired mo- 


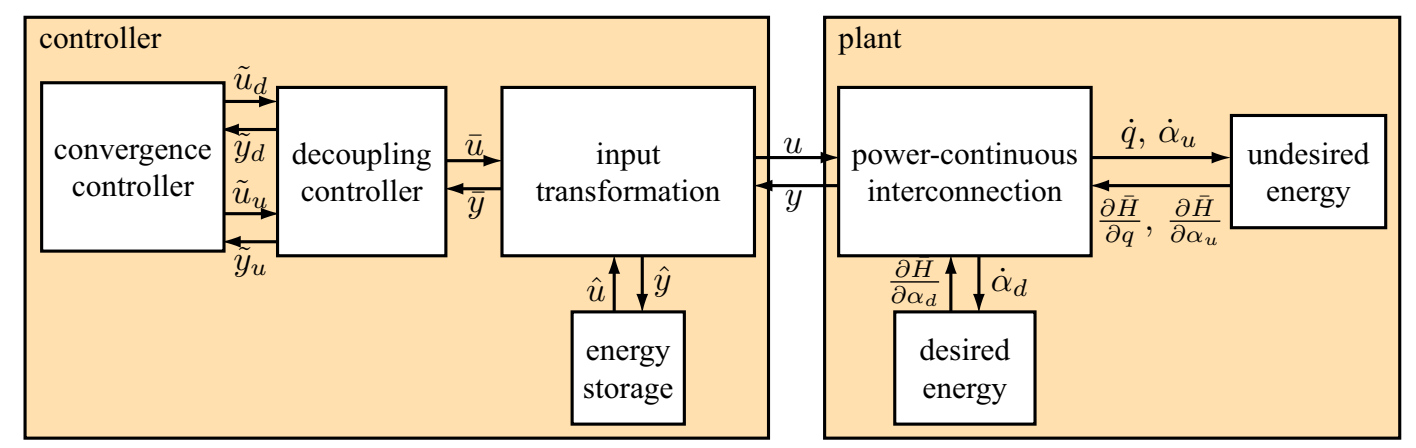

Fig. 1. Block diagram representation of the interconnection of the complete controller with the plant.

menta for the plant interconnected to the subcontrollers (6)-(8) as

$$
\begin{aligned}
& \dot{\alpha}_{d}=S_{d} \frac{\partial \bar{H}}{\partial \alpha_{d}} \\
& \dot{\alpha}_{u}=S_{u} \frac{\partial \bar{H}}{\partial \alpha_{u}}
\end{aligned}
$$

which shows how the term $X$ in (9) has canceled the original coupling between $\alpha_{d}$ and $\alpha_{u}$. So with these sub-controllers interconnected, both the desired and undesired energy are conserved. As an extra design freedom, the skew-symmetric matrices $S_{d}$ and $S_{u}$ can be chosen freely, for example to minimize the required control torques.

Finally, the sub-controller (10) can be shown to establish a unidirectional flow of energy from the undesired part to the desired part until $\alpha_{u}$ is zero, i.e., until the system moves along the desired distribution.

The interesting properties of the sub-controllers can be seen from their formulation in portHamiltonian form: sub-controllers (6), (8) and (10) are power-continuous, meaning they only redistribute the energy; they do not dissipate, store, or create energy in the system.

\section{APPLICATION TO WALKING ROBOTS}

\subsection{Dynamic model of a compass-gait biped}

We now show how to modify and apply the results of Section 3 to the planar compass-gait biped shown in Fig. 2. This biped is studied in detail in (Goswami et al., 1997), and we use the same parameters here.

We have modeled the biped as a port-Hamiltonian system of the form (2) using coordinates $q_{1}$ through $q_{4}$ for the positions and another four coordinates for the momenta. Impacts of the feet with the ground are assumed to be instantaneous and inelastic. This means that on impact, the momentum of the robot's foot along the direction of the vertical and horizontal ground forces is reset,

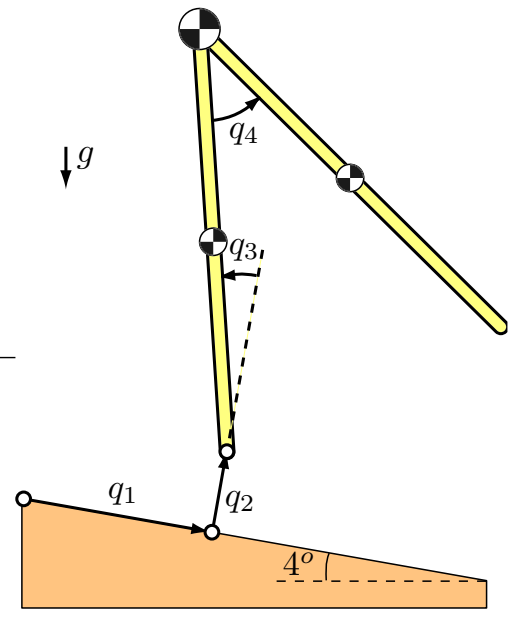

Fig. 2. Schematic of the compass-gait biped.

causing an instantaneous loss of (some) kinetic energy. Then, during the contact phase, the ground reaction forces are computed and applied to the robot, provided that the required vertical force is positive, i.e., the ground is pushing upward.

Although this model is suitable to model general motions of the biped (including flight-phase), it is useful to simplify the model for control purposes. Namely, during normal walking with one foot on the ground, and assuming the ground reaction forces are positive, the biped can be modeled as a two-link world-fixed mechanism. By using either the coordinates $q_{3}$ and $q_{4}$ or the coordinates $q_{3}+q_{4}$ and $-q_{4}$ (depending on which foot is on the floor), a single dynamic model can be used for both situations (since the biped is symmetric). We use this reduced model to design the controller, and we assume we can apply a control torque both at the hip and at the ankle of the leg that is touching the ground. This means that, under the conditions that the reduced model is valid, we have a fully actuated robot.

\subsection{Nominal passive compass-gait walking}

When the biped is placed on a shallow slope (we chose a $4^{\circ}$ slope) and given the right initial conditions, it can walk stably downhill without 


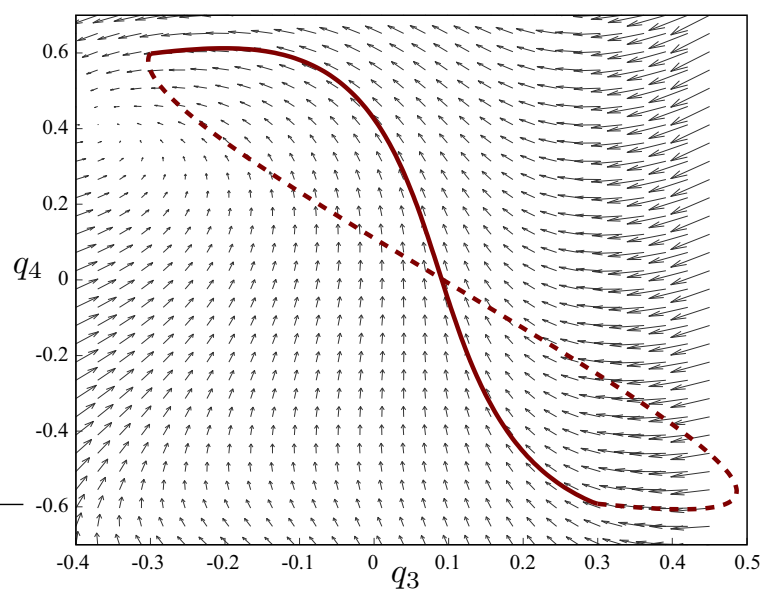

Fig. 3. Nominal trajectory and desired vector field for the compass-gait biped. The solid line represents the stance phase, the dashed line the swing phase.

actuation. This has been shown before both in simulations and experiments, see e.g. (McGeer, 1990; Garcia et al., 1998; Goswami et al., 1997). The biped gains energy from the gravitational field, but looses energy due to the inelastic impacts of the feet with the ground.

The gait of the biped (i.e., the cyclic motion of some of its configuration variables) is represented in Fig. 3 as a closed curve, consisting of a solid part and a dashed part, each corresponding to one leg being on the ground and the other swinging. The transition between the two parts occurs when the front leg touches the ground (causing a liftoff of the rear leg): the instantaneous change of momentum due to the impact can be seen in the figure as a non-smoothness in the curve.

This gait is stable in the sense that for small perturbations in the initial conditions, as well as for small disturbances, the motion converges to the same gait and the biped does not fall. However, its region of attraction is quite small; a slightly larger perturbation causes the biped to fall over. This is shown in the top figure of Fig. 4: the biped falls when walking down a small step. Simulation showed that any drop larger than $0.5 \%$ of the leg length results in the robot falling over.

\subsection{Controlled passive compass-gait walking}

In this section, we apply the results of Section 3 and design a port-based controller that enlarges the region of attraction of the passive walker and thus makes it more robust against disturbances.

Since we can only apply a control torque to the ankle when the leg is touching the ground, we design a control law for the stance phase of the leg only. When the front leg touches the ground (and simultaneously the rear leg lifts off), we switch control to the other ankle and apply the same control law symmetrically.

The control goal is to augment the existing passive walking and not alter the basic behavior of the robot, hence we take the nominal uncontrolled trajectory as the desired trajectory and construct a suitable vector field around it, as shown in Fig. 3. The vectors of the field are tangent to the nominal trajectory (during the stance phase) or are directed along and towards it. For this simple example, the vector field was generated by fitting polynomial descriptions for the two components of the vector field to several desired integral curves.

With this vector field chosen, we then apply the control laws (6), (8), and (10). However, instead of (7), we choose a different approach. The reason is that the potential energy (gravity) in the system is partially responsible for shaping the nominal trajectory: to compensate for the potential energy would make the trajectory unnatural and hence would require extra control effort. So instead of using (7), we only compensate for the potential energy in the undesired directions, i.e., in the directions along $\alpha_{u}$, or orthogonal to the vector field:

$$
\hat{u}=h_{u}\left(h_{u}^{T} M^{-1} h_{u}\right)^{-1} h_{u}^{T} M^{-1} \frac{\partial V}{\partial q}
$$

A disadvantage of this solution, however, is that it is not passive. Therefore, we monitor the energy injected to or removed from the system through the port $(\hat{u}, \hat{y})$ and apply an extra torque in the desired direction of motion that dissipates or injects this energy back in the system.

The resulting controller is a port-interconnection of power-continuous sub-controllers, together with the compensation for potential energy. When the system is moving along the nominal trajectory, it does not apply a control torque, but when it deviates from the trajectory, the controller exerts the required torques to get back to the nominal trajectory. The performance is shown in the lower figure of Fig. 4, where the biped can be seen to successfully walk down a step: when the front leg touches the ground at a lower point than before, the controller quickly drives the rear leg forward (towards the nominal trajectory) preventing the biped from falling forward, which occurs in the uncontrolled case.

Fig. 5 shows a plot of the various energies involved in the system. The total energy can be seen to be constant except during impacts (when there is a jump) and when the control energy is (temporarily) large, after the step down around $t=6.5 \mathrm{~s}$. During nominal walking, potential and desired kinetic energy are exchanged, while the undesired kinetic energy and the control energy stay low. 

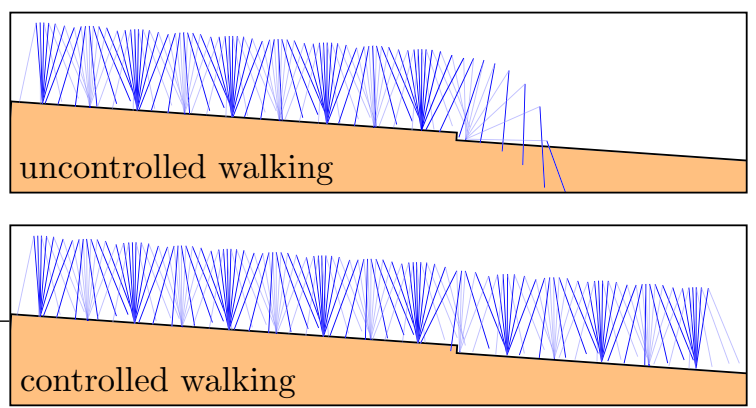

Fig. 4. Stick diagrams of uncontrolled and controlled walking down a step.

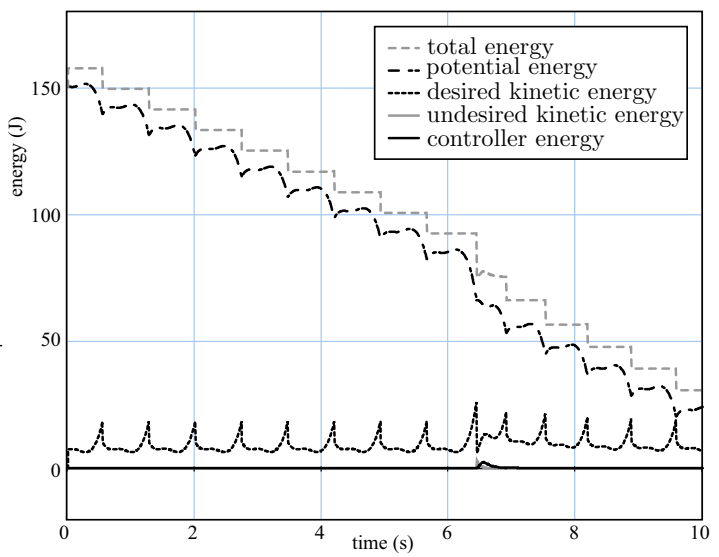

Fig. 5. Energy plot of the controlled walk of Fig. 4. The step down occurs around $t=6.5 \mathrm{~s}$.

\section{CONCLUSIONS AND FUTURE WORK}

We described a general port-based technique of modular controller design for fully actuated mechanical systems, and demonstrated how this method can be used to increase the robustness of a compass-gait passive bipedal robot against unknown disturbances. The resulting controller does not alter the nominal unactuated passive gait of the biped, but provides corrective control action when the biped deviates from the nominal trajectory and thus prevents it from falling over. We showed in simulation how, in this way, the biped can walk down a step without falling over like in the uncontrolled case.

In future work, we want to apply the same method to more elaborate walking robots (e.g. having knees or moving in three dimensions) and test the method on experimental setups.

Furthermore, we want to extend the idea to timevarying vector fields, where the vector field (and the corresponding nominal trajectory) is optimized for various walking speeds, slopes, foot clearance, or other aspects of practical walking. Depending on the higher-level task the robot has to perform, the right vector field is chosen and the lower-level controller then makes the robot move along that vector field.
Finally, we want to study the trajectories themselves in order to be able to compute the 'optimal' trajectories for certain robots in certain situations from scratch, without the need for simulation or numerical searches for a stable limit cycle.

\section{ACKNOWLEDGMENTS}

This work has been done in the context of the European sponsored project GeoPlex with reference code IST-2001-34166. Further information is available at http://www.geoplex.cc.

\section{REFERENCES}

Blankenstein, G. (2000). Implicit Hamiltonian Systems: Symmetry and Interconnection. $\mathrm{PhD}$ thesis. University of Twente.

Collins, S.H., M. Wisse and A. Ruina (2001). A Three-Dimensional Passive-Dynamic Walking Robot with Two Legs and Knees. International Journal of Robotics Research 20(7), 607-615.

Duindam, V. and S. Stramigioli (2005). PortBased Asymptotic Curve Tracking for Mechanical Systems. to appear in European Journal of Control.

Garcia, M., A. Chatterjee, A. Ruina and M. Coleman (1998). The Simplest Walking Model: Stability, Complexity, and Scaling. ASME Journal of Biomechanical Engineering 120(2), 281-288.

Goswami, A., B. Espiau and Keramane A. (1997). Limit cycles in a passive compass gait biped and passivity-mimicking control laws. Journal of Autonomous Robots 4(3), 273-286.

Li, P.Y. and R. Horowitz (1995). Passive Velocity Field Control of Mechanical Manipulators. In: Proceedings of the IEEE International Conference on Robotics and Automation. pp. 2764-2770.

Li, P.Y. and R. Horowitz (1999). Passive Velocity Field Control of Mechanical Manipulators. IEEE Transactions on Robotics and Automation 15(4), 751-763.

McGeer, T. (1989). Powered flight, child. In: Proceedings of the IEEE International Conference on Robotics and Automation. Vol. 3. pp. $1592-1597$.

McGeer, T. (1990). Passive Dynamic Walking. The International Journal of Robotics Research $\mathbf{9}(2), 62-82$.

van der Schaft, A.J. (2000). L $L_{2}$-Gain and Passivity Techniques in Nonlinear Control. Communications and Control Engineering. SpringerVerlag. 